\title{
Seguimento de Caminhos Visuais: Avaliação de Desempenho de uma Estratégia Baseada em NMPC para Robôs Diferenciais
}

\author{
Tiago T. Ribeiro* Ramon O. Fernandez* \\ André Gustavo S. Conceição* \\ * LaR - Laboratório de Robótica, Departamento de Engenharia \\ Elétrica, Universidade Federal da Bahia, Salvador, Bahia, Brasil \\ (e-mails: tiagotr@ufba.br, ramon.elt.ba@gmail.com, \\ andre.gustavo@ufba.br).
}

\begin{abstract}
Robot control strategies based on computer vision allow to increase the range of potential applications. However, before made available in a real scenario, it is necessary to fully characterize the main functionalities. This paper presents performance evaluations of a strategy for NMPC-based visual path following control for differential robots. More specifically, we study the influence of the control and prediction horizons, parameters that directly interfere with the feasibility and stability requirements, and analyze the performance of controllers when there are high tracking errors. Experimental results demonstrate the benefits of three modalities of NMPC controllers for an arbitrary, closed and high-length path.

Resumo: Estratégias de controle para robôs móveis baseadas em visão computacional permitem aumentar a gama de aplicações potenciais. Porém, antes da sua disponibilização num cenário real, necessita-se de completa caracterização das principais funcionalidades. Este artigo objetiva avaliar o desempenho de uma estratégia para o seguimento de caminhos visuais baseada em NMPC para robôs diferenciais. Mais especificamente, estuda-se a influência dos horizontes de controle e predição, parâmetros que interferem diretamente nos requisitos de factibilidade e estabilidade, além de analisar o desempenho dos controladores quando existem erros de rastreamento elevados. Resultados experimentais demonstram os benefícios de três modalidades de controladores NMPC para um caminho arbitrário, fechado e de comprimento elevado.
\end{abstract}

Keywords: Mobile Robotics; Path Following; Predictive Control; Visual Control; Computer Vision.

Palavras-chaves: Robótica móvel; Seguimento de Caminho; Controle Preditivo; Controle Visual; Visão computacional.

\section{INTRODUÇÃO}

Recentemente, com o objetivo de obter soluções para navegação robótica autônoma de alta precisão, grandes esforços tem sido dedicados ao desenvolvimento de estratégias de controle baseadas em visão computacional, tal como em Zhang et al. (2017) e Ke et al. (2018), com ênfase especial dada a soluções baseadas em controle servo visual.

Para incrementar a capacidade de navegação em algumas aplicações reais, essas soluções são ajustadas para lidar com múltiplas imagens de referência (Araar et al. (2017); Rodrigues et al. (2018)), característica intrínseca de aplicações como seguimento do caminhos contínuos. Neste caso, as abordagens para controle servo visual tradicionais (PBVS e IBVS) tornam-se computacionalmente ineficientes devido a necessidade de calcular a inversa da matriz de iteração para um grande número de características visuais.

No caso de seguimento de caminhos para robôs móveis, o objetivo é minimizar os erros obtidos de características visuais simplificadas, sendo a lei de controle calculada diretamente do plano da imagem. Tal requerimento de sim- plicidade surge da necessidade de manter baixos índices de complexidade computacional especialmente para sistemas com dinâmicas rápidas e não lineares.

Na presente década, muitas soluções foram propostas, tal como em Das et al. (2010) com base na odometria visual de um sistema multi-câmera e na correspondência de pontos visuais de referência, v. Es and Barfoot (2015) através de uma proposta de estrutura de grafos arbitrariamente conectados e em Huang et al. (2016) tratando o seguimento de caminho como um problema de regressão cujos modelos são obtidos através da Programação de Expressão Genética. Porém, em todos os casos, estas propostas não tratam explicitamente dos limites impostos pelos sistemas visuais, sendo deste modo uma boa oportunidade para aplicação de estratégia de controle ótimo.

Neste contexto, Ribeiro (2016b) propõe a aplicação de NMPC (do inglês, Nonlinear Model Predictive Control) para a resolução do problema de seguimento de caminhos visuais diretamente no plano da imagem para robôs diferenciais comerciais. Problemas intrínsecos da plataforma experimental, especialmente associados aos sistemas visual 
e eletromecânico motivaram a busca de soluções para garantia de factibilidade e estabilidade, sendo produzidos os resultados apresentados em Ribeiro (2016a). Ribeiro and Scolari Conceicao (2018) fornece um comparativo analítico entre três modalidades de controladores NMPC e estuda a influência da velocidade de navegação, grandeza estritamente relacionada ao desemenho do sistem visual. Tais resultados evidenciam a necessidade de se avaliar o desempenho em outras condições de funcionamento e variabilidade de parâmetros de sintonia dos controladores NMPC, para que se possa garantir aplicabilidade prática em situações reais, tais como para movimentação de peças na indústria ou sistemas de transporte.

Este artigo objetiva avaliar o desempenho da estratégia baseada em controladores NMPC supracitada com relação a variação dos horizontes e erros de rastreamento. Tais controladores são implementados e resultados experimentais demonstram melhor desempenho da estratégia com garantia de factibilidade. Com este estudo espera-se aumentar o grau de confiabilidade da proposta.

O restante deste artigo está organizado da seguinte forma: A Seção 2 apresenta os modelos e as estratégias de controle. A Seção 3 apresenta alguns detalhes da plataforma experimental. Os resultados obtidos são apresentados na Seção 4 e as considerações finais são fornecidas na Seção 5 .

\section{MODELOS E CONTROLADORES}

\subsection{Seguimento de Caminhos visuais}

A Figura 1 ilustra os elementos necessários à formulação do problema de seguimento de caminhos visuais conforme proposto em Ribeiro (2016b).

Por conveniência, replica-se aqui o seguinte modelo cinemático:

$$
\dot{\mathbf{x}}_{e}=f\left(\mathbf{x}_{e}, \mathbf{u}_{e}\right)=\left[\begin{array}{c}
\dot{Z} \\
\dot{\theta_{r}}
\end{array}\right]=\left[\begin{array}{c}
\omega H+(\omega Z+v) \tan \left(\theta_{r}\right) \\
\mathbf{u}_{e}
\end{array}\right] .
$$

sendo:

$Z$ : Espaçamento lateral entre o robô e o caminho;

$\theta_{r}$ : Erro angular entre o eixo longitudinal ao robô e a reta tangente ao caminho;

$v$ Velocidade linear;

$\omega$ Velocidade angular;

$H$ : Horizonte para avaliação do caminho no plano da imagem;

$\mathbf{u}_{e}$ : Ação de controle.

Com as saídas sendo os próprios estados, o problema de controlar os movimentos dos robôs diretamente do plano da imagem, pode ser sumarizado da seguinte forma:

\section{Encontrar $\omega$, tal que $Z$ e $\theta_{r}$ sejam factíveis.}

Um etapa particularmente importante para o sucesso da implementação deste método é o cálculo da curvatura do caminho visual $c(s)$. Como $Z$ e $\theta_{r}$ são parâmetros obtidos são estimativas aproximadas, sem requerimentos rigorosos na calibração do sistema visual, qualquer erro nessas medições se propagará através do cálculo $c(s)$, não garantindo assim completa precisão.
Neste trabalho, tal curvatura é obtida com razoável precisão através do uso de 2 pontos na vizinhança ponto principal da imagem e calculando o raio do círculo passando pelos três pontos. Vale ressaltar que a precisão dos movimentos dos robôs ao longo do caminho visual será de grande relevância para o cálculo das curvaturas visuais.

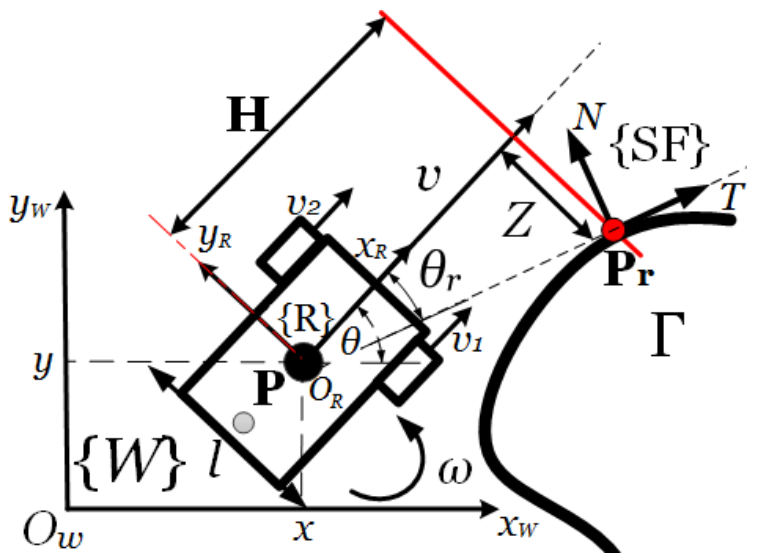

(a) Sistemas de coordenadas

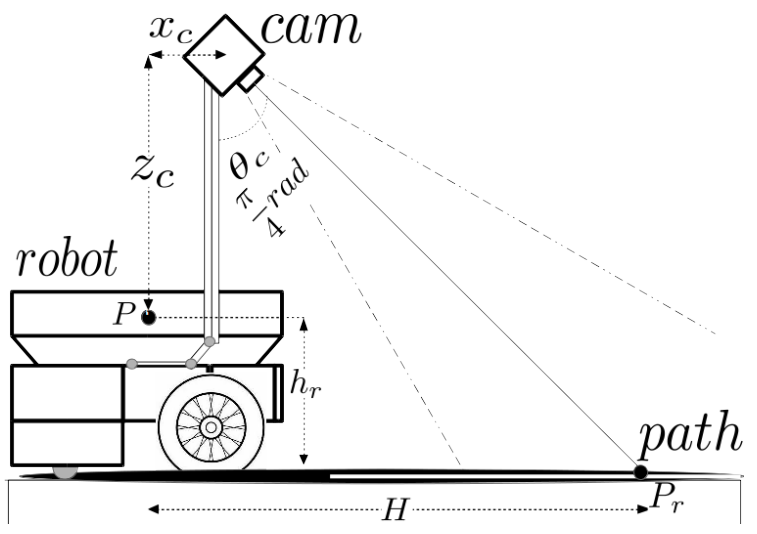

(b) Vista lateral.

Figura 1. Seguimento de caminhos visuais.

\subsection{Controladores $N M P C$}

Os controladores NMPC com garantia de estabilidade utilizados neste trabalho baseiam-se na inclusão de custo e restrições terminais, proposta por Mayne and J. B. Rawlings (2000), e possuem a seguinte formulação geral:

$$
\begin{array}{r}
J_{\text {min }}=\min _{\mathbf{u}_{e}} \int_{t}^{t+T_{p}} \quad F\left(\mathbf{x}_{e}(\tau), \mathbf{u}_{e}(\tau)\right) d \tau+V\left(\mathbf{x}_{e}\left(t+T_{p}\right)\right), \\
\text { sujeito a: } \dot{\mathbf{x}}_{e}(\tau)=f\left(\mathbf{x}_{e}(\tau), \mathbf{u}_{e}(\tau)\right), \\
\mathbf{u}_{e}(\tau) \in \mathcal{U}, \forall \tau \in\left[t, t+T_{c}\right], \\
\mathbf{x}_{e}(\tau) \in \mathcal{X}, \forall \tau \in\left[t, t+T_{p}\right], \\
\mathbf{x}_{e}\left(t+T_{p}\right) \in \Omega,
\end{array}
$$

sendo o custo de etapa $F$ dado por:

$$
F\left(\mathbf{x}_{e}(\tau), \mathbf{u}_{e}(\tau)\right)=\mathbf{x}_{e}^{T} \mathbf{Q} \mathbf{x}_{e}+\mathbf{u}_{e}^{T} \mathbf{R} \mathbf{u}_{e}
$$

sendo:

$T_{p}$ : Horizonte de predição;

$T_{c}$ Horizonte de controle; com $T_{c} \leq T_{p}$; 
$\mathcal{U}$ : Conjunto de entradas factíveis;

$\mathcal{X}$ Conjunto de estados factíveis;

Q, R: Matrizes positivas definidas que ponderam os desvios nos estados e entradas, respectivamente;

$V(\bullet)$ : Custo terminal;

$\Omega$ : Conjunto das restrições terminais.

Para uma função de Lyapunov $V\left(\mathbf{x}_{e}\left(t+T_{p}\right)\right)=\frac{1}{2} \mathbf{x}_{e}(t+$ $\left.T_{p}\right)^{T} \mathbf{P} \mathbf{x}_{e}\left(t+T_{p}\right)$, após manipulação pertinente, tem-se a seguinte condição de estabilidade:

$$
\begin{array}{r}
\alpha p_{22}-q_{22}-r_{11} \alpha^{2} \geq q_{11} ; \\
\left|\theta_{r_{T}}\right| \geq\left|Z_{T}\right| \\
p_{11} Z_{T}\left(\omega H+\left(\omega Z_{T}+v\right) \tan \theta_{r_{T}}\right) \leq 0 .
\end{array}
$$

A região $\Omega$ é adicionalmente limitada pelas ações de controle terminais, da seguinte forma:

$$
\omega=\frac{c v-\alpha \theta_{r_{T}} \cos \theta_{r_{T}}}{\cos \theta_{r_{T}}-c Z_{T}},
$$

$\operatorname{com} \omega$ restrito aos limites praticáveis no robô:

$$
\omega_{\min } \leq \omega \leq \omega_{\max }
$$

O algoritmo NMPC sem garantia de estabilidade é implementado neste trabalho anulando o custo terminal $V\left(\mathbf{x}_{e}\left(t+T_{p}\right)\right)$ e fazendo $\Omega=\mathbb{R}$

Para garantia de factibilidade, adota-se um esquema de relaxamento das restrições com posterior saturação dos esforços de controle. Mais especificamente, utiliza-se a seguinte política para as restrições dos estados:

$$
\begin{aligned}
\mathbf{x}^{\text {bound }}= & \mathrm{x}_{\mathbf{e}}^{\max }+\mathrm{x}_{\text {tol }} ; \\
& \mathrm{x}^{\text {bound }} \in \mathcal{X},
\end{aligned}
$$

com $\mathbf{x}_{\mathbf{e}}^{\max }$ o vetor de erros de estado máximos ao longo do horizonte de predição e $\mathbf{x}_{\text {tol }}$ um vetor de tolerâncias constantes para cada estado. Além disto, admite-se que as entradas otimizadas sejam elevadas o suficiente para a manutenção da factibilidade recursiva. A ideia principal do método é sumarizada no Algoritmo 1.

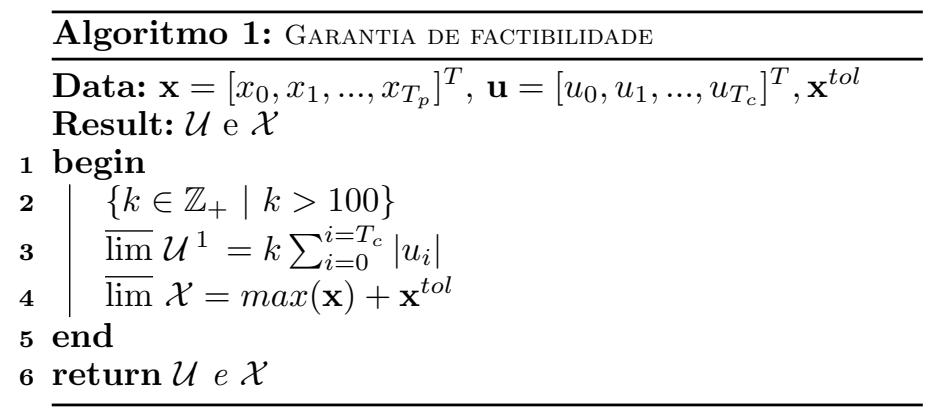

Com este tratamento da factibilidade, sub-otimalidade proposta por Scokaert et al. (1999) pode ser utilizada como estratégia para garantia de estabilidade, desde que, com esta abordagem, factibilidade implica em estabilidade.

Este tratamento das restrições nos estados é de certa forma similar à abordagem denominada por restrições brandas (do inglês, "soft-constraint") de Oliveira and Biegler (1994), porém sem qualquer penalização explícita

\footnotetext{
$\overline{1 \varlimsup} \mathcal{S}$ denota o limite superior do conjunto $\mathcal{S}$
}

na função objetivo, mantendo-se baixas especificações de complexidade computacional.

Tal proposta é utilizada para seguimento de caminhos com elevados erros de rastreamento que tendem a ocupar o processamento de tal forma que novas imagens não sejam adquiridas, causando instabilidade.

\section{PLATAFORMA EXPERIMENTAL}

O robô diferencial utilizado (Figura 2(a)) foi construído com a plataforma Lego ${ }^{\circledR}$ Mindstorms ${ }^{\circledR} E V 3$ e possui uma webcam Microsoft ${ }^{\circledR}$ LifeCam HD-3000 com a seguinte pose (relativa a $\{\mathrm{R}\}): x_{c}=0,05 \mathrm{~m} ; y_{c}=0,05 \mathrm{~m} ; z_{c}=$ $0,2 \mathrm{~m} ; \theta_{c}=\frac{\pi}{4} \mathrm{rad}$.

As análises foram feitas em situações onde a função de curvatura é variável, especificamente para o caminho arbitrário, com cerca de 8 metros de comprimento, ilustrado na Figura 2(b). Como pode ser visto, o perfil de curvatura variável tende a exigir mais das ações de controle em diversos trechos.

O sistema visual foi calibrado considerando os seguintes valores limitantes para os erros de estado (limites do campo visual da câmera): $Z= \pm 0.1 \mathrm{~m} ; \Theta_{r}= \pm 0.8 \mathrm{rad}$. Os problemas de otimização foram resolvidos através do pacote livre DONLP2 Spellucci (1998), um otimizador não linear de propósito geral para variáveis contínuas que implementa programação quadrática sequencial com uma técnica de conjuntos ativos.

O esquema de controle utilizado baseia-se numa estrutura em cascata, onde um laço interno controla as velocidades dos motores através do algoritmo PID clássico e cujas referências são fornecidas por um laço externo que implementa o NMPC visual. Tais referências, são os esforços de controle físicos deste laço externo $(v, \omega)$ e são obtidos através da entrada de controle ótima, $u\left(t_{k}\right)$ ótima a cada instante de amostragem, definido como sendo $T_{s}=0,2 \mathrm{~s}$.

\section{RESULTADOS EXPERIMENTAIS}

Nos experimentos seguintes, as estratégias NMPC sem garantias (modo N), com garantia de estabilidade (modo GE) e com garantia de factibilidade (modo GF), foram avaliadas considerando os seguintes parâmetros de sintonia:

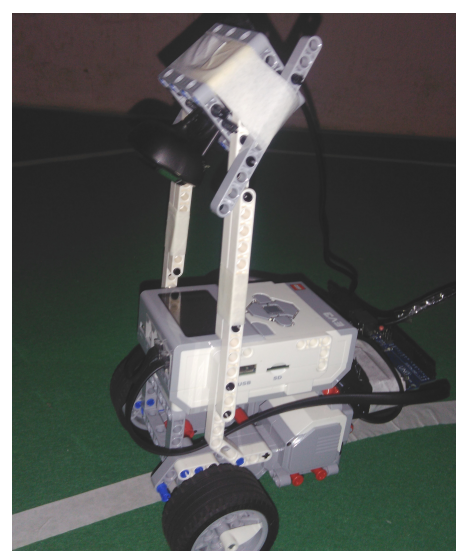

(a) Robô móvel.

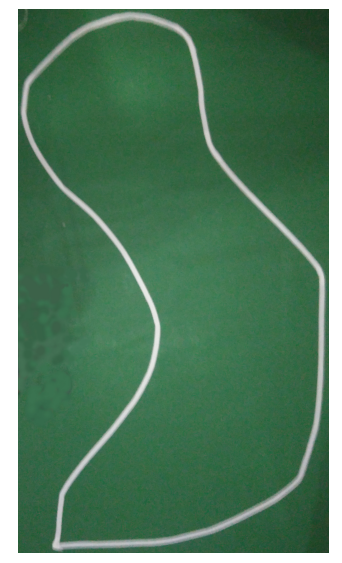

(b) Caminho.
Figura 2. Plataforma experimental. 
$\mathbf{Q}_{\mathbf{i}}=\operatorname{diagonal}(10 ; 1) ; \mathbf{R}_{\mathbf{i}}=0,2 ; P=\operatorname{diagonal}(100 ; 100) ;$ $\alpha=2$. As restrições elementares foram definidas como segue:

$$
\left|u_{1}\right| \leq 0,2 a \mathrm{~m} / \mathrm{s} ;|z| \leq 0,05 \mathrm{~m} ;\left|\theta_{e}\right| \leq 0,3 \mathrm{rad}
$$

Adicionalmente, para o modo GE, foram utilizadas as condições definidas em (8)-(12) e para o modo GF implementa-se o Algoritmo 1.

\subsection{Influência do horizontes}

Nesta etapa, os experimentos são feitos variando os horizontes de predição $T_{p}$ e de controle $T_{c}$. Inicialmente, objetiva-se analisar como o aumento de tais horizontes influenciam no tempo de processamento $T_{\text {proc }}$.

Sabe-se que tal medida é influenciada pela geometria do caminho, velocidade de navegação, parâmetros de sintonia e plataforma computacional, sendo portanto, os testes realizados para o caso em que todas as abordagens apresentaram desempenho razoável de acordo com Ribeiro and Scolari Conceicao (2018), mais especificamente $v=0,1 \mathrm{~m} / \mathrm{s}$. Tais análises são importantes para nortear a escolha da abordagem mais adequada em determinada aplicação, além de ratificar a necessidade da utilização de valores pequenos para os horizontes, haja vista a não linearidade do modelo.

A Tabela 1 fornece os dados obtidos. Observa-se que modo GF apresenta melhores resultados, uma vez que as medidas de $T_{\text {proc }}$ foram mais uniformes ao longo do intervalo de análise e menores valores de variância indicam que amostras não foram perdidas. Isto se deve as folgas acrescentadas nas restrições, o que permite diminuir o tempo de cálculo das ações de controle, sendo possível a aquisição de novas imagens que são utilizadas para a correção dos possíveis erros oriundos das ações de controle anteriormente saturadas. É possível concluir também a viabilidade da definição de $T_{p}=T_{c}=3 T_{s}$ para os experimentos de avaliação de erros de rastreamento iniciais elevados, na próxima subseção.

Tabela 1. Influência dos horizontes

\begin{tabular}{|c|c|c|c|}
\hline modo & $T_{p}=T_{c}$ & $T_{\text {proc }}$ & $\operatorname{var}\left(T_{\text {proc }}\right)$ \\
\hline \multirow{4}{*}{ N } & 1 & 4,595 & 0,898 \\
\cline { 2 - 4 } & 3 & 4,800 & 0,567 \\
\cline { 2 - 4 } & 5 & 7,1702 & 1,396 \\
\cline { 2 - 4 } & 7 & 5,132 & 114,013 \\
\cline { 2 - 4 } & 15 & 39,092 & $4,312 \mathrm{e}+04$ \\
\hline \multirow{4}{*}{ GE } & 1 & 4,768 & 2,206 \\
\cline { 2 - 4 } & 3 & 5,074 & 2,764 \\
\cline { 2 - 4 } & 5 & 7,066 & 782,469 \\
\cline { 2 - 4 } & 7 & 9,071 & $1,275 \mathrm{e}+03$ \\
\hline \multirow{4}{*}{ GF } & 15 & 41,541 & $4,180 \mathrm{e}+04$ \\
\cline { 2 - 4 } & 1 & 4,545 & 0,427 \\
\cline { 2 - 4 } & 3 & 5,513 & 121,643 \\
\cline { 2 - 4 } & 7 & 7,072 & 758,819 \\
\cline { 2 - 4 } & 15 & 7,307 & 886.876 \\
\hline
\end{tabular}

Para ratificar este resultado, foram utilizadas duas métricas quantitativas, mais especificamente, utiliza-se a integral do erro absoluto $(I A E)$ e a variação de controle total $(T V)$. O índice $I A E$, calculado por $\int_{0}^{T_{E N D}}|e(t)| d t$, é muito utilizado para comparação de desempenho de estratégias distintas em experimentos semelhantes, por outro lado o índice $T V$, calculado por $\sum_{k=0}^{k_{E N D}}|u(k)-u(k-1)|$, objetiva avaliar o efeito dos ruídos nos sinais de controle.

Para o modo N, a Tabela 2 apresenta valores bem próximos a menos do horizonte máximo avaliado $\left(T_{P}=T_{C}=15\right)$ que apresentou valores elevados.

Com a garantia de estabilidade, a Tabela 3 mostra pequeno aumento de ambos os índices com o aumento das ações de controle, na maioria dos casos, indicando redução da capacidade de regulação e rejeição de ruído quando se aumentam os horizontes, comportamento justificado pelo aumento da quantidade de variáveis a serem atraídas para a região terminal das restrições, além do efeito do custo terminal, que deterioram o custo computacional.

Para a garantia de factibilidade, a Tabela 4 mostra maior uniformidade dos índices de desempenho, independente dos horizontes adotados, indicando capacidade superior de regulação dos erros de estado com baixas variações nos esforços de controle.

Tabela 2. Análise de desempenho - modo N

\begin{tabular}{|c|c|c|c|c|}
\hline \hline & \multicolumn{2}{|c|}{$\mathrm{IAE}$} & \multicolumn{2}{c|}{$\mathrm{TV}$} \\
\hline$T_{p}=T_{c}$ & $z_{I A E}[\mathrm{~m}]$ & $\theta_{r_{I A E}}[\mathrm{rad}]$ & $v_{T C V}[\mathrm{~m} / \mathrm{s}]$ & $\omega_{T C V}[\mathrm{rad} / \mathrm{s}]$ \\
\hline \hline 1 & 1,022 & 6,567 & 0,827 & 13,040 \\
\hline 3 & 1,015 & 6,490 & 0,815 & 15,798 \\
\hline 5 & 1,047 & 6,760 & 0,865 & 13,872 \\
\hline 7 & 1,031 & 6,642 & 0,891 & 15,673 \\
\hline 10 & 1,045 & 6,730 & 0,859 & 13,995 \\
\hline 15 & 1,849 & 10,712 & 1,654 & 16,961 \\
\hline \hline
\end{tabular}

Tabela 3. Análise de desempenho - modo GS

\begin{tabular}{|c|c|c|c|c|}
\hline \hline & \multicolumn{2}{|c|}{$\mathrm{IAE}$} & \multicolumn{2}{c|}{$\mathrm{TV}$} \\
\hline$T_{p}=T_{c}$ & $z_{I A E}[\mathrm{~m}]$ & $\theta_{r_{I A E}}[\mathrm{rad}]$ & $v_{T C V}[\mathrm{~m} / \mathrm{s}]$ & $\omega_{T C V}[\mathrm{rad} / \mathrm{s}]$ \\
\hline \hline 1 & 1,108 & 7,297 & 0,842 & 16,478 \\
\hline 3 & 1,101 & 7,228 & 0,877 & 18,918 \\
\hline 5 & 1,025 & 6,661 & 0,859 & 13,922 \\
\hline 7 & 1,043 & 6,808 & 0,835 & 14,438 \\
\hline 10 & 1,017 & 6,643 & 0,875 & 14,660 \\
\hline 15 & 1,309 & 8,150 & 1,153 & 16,319 \\
\hline \hline
\end{tabular}

Tabela 4. Análise de desempenho - modo GF

\begin{tabular}{|c|c|c|c|c|}
\hline \hline & \multicolumn{2}{|c|}{$\mathrm{IAE}$} & \multicolumn{2}{c|}{$\mathrm{TV}$} \\
\hline$T_{p}=T_{c}$ & $z_{I A E}[\mathrm{~m}]$ & $\theta_{r_{I A E}}[\mathrm{rad}]$ & $v_{T C V}[\mathrm{~m} / \mathrm{s}]$ & $\omega_{T C V}[\mathrm{rad} / \mathrm{s}]$ \\
\hline \hline 1 & 1,093 & 7,046 & 0,983 & 14,245 \\
\hline 3 & 1,111 & 7,139 & 0,919 & 13,292 \\
\hline 5 & 1,100 & 7,178 & 0,985 & 13,564 \\
\hline 7 & 1,080 & 6,989 & 0,889 & 13,548 \\
\hline 10 & 1,117 & 7,191 & 0,867 & 13,684 \\
\hline 15 & 1,119 & 7,262 & 0,901 & 13,486 \\
\hline
\end{tabular}

\subsection{Erros no caminho visual}

Objetiva-se aqui analisar o desempenho dos controladores NMPC na presença de erros de rastreamento elevados, podendo ser causados, por exemplo, por falhas momentâneas no sistema visual. Em tal análise, posiciona-se o robô em relação ao caminho para que existam dois tipos de erros visuais: Caminho visto como uma linha vertical à direita do ponto principal e como uma linha praticamente horizontal 
próximo ao horizonte. Estes testes são particularmente importantes para verificar, por exemplo, como se comportam as estratégias quando o robô chega ao fim do caminho, mesmo sem um tratamento matemático específico para a descontinuidade verificada neste ponto.

A Figura 3 ilustra os resultados para linha vertical. Observa-se que, para para os modos N e GE, os erros são regulados momentaneamente, porém, nos instantes posteriores os erros crescem no sentido oposto até a perda total do caminho do campo visual. Para o modo GF, os erros são regulados satisfatoriamente. Deste modo, demonstra-se em termos práticos, a importância da factibilidade recursiva para a garantia de estabilidade.

Na Figura 4 verifica-se que, para este caso em que existe uma certa tendência de seguimento para o caminho visual (inclinação na direção de seguimento), todos os controladores conseguiram regular os erros de seguimento com esforços de controle satisfatórios. Neste caso, o modo GF apresentou regulação mais suave e num intervalo de tempo menor. É válido ressaltar que os testes foram realizados em condições próximas das ideais de iluminação e cadência visual, de modo que, maiores investigações devem ser realizadas relativamente à robustez do método.

\section{CONSIDERAÇÕES FINAIS}

Este trabalho apresentou análises de desempenho de uma estratégia para seguimento de caminhos visuais, baseadas em NMPC, para robôs diferenciais, objetivando-se caracterizar as funcionalidades principais antes da aplicação em cenários reais. Foram realizados testes experimentais com uma plataforma construída com elementos comerciais, o que certamente é um atrativo para potenciais utilizadores das técnicas.

Foram feitas avaliações quando se variam os horizontes de predição e controle, ocasião em que se confirmou a inviabilidade da escolha de valores elevados nos modos $\mathrm{N}$ e GE. Destaca-se mais uma vantagem do modo GF, já que os tempos de processamento permaneceram aproximadamente constantes, independente do horizonte escolhido. Ainda nesta etapa, os critérios de desempenho $I A E$ e $T V$ evidenciaram a superioridade da desta abordagem e isto se deve à maneira como as restrições são relaxadas, sem penalização adicional na função objetivo.

Finalmente, foram feitas análises para o seguimento de caminhos visuais com erros de rastreamento elevados, sendo, mais uma vez, confirmado melhor desempenho dos controladores NMPC com factibilidade garantida. Tal análise é de fundamental importância para o tratamento de descontinuidades no caminho visual, situação que ocorre ao final dos percurso escolhido.

Trabalhos futuros envolvem a obtenção de um novo modelo e controle NMPC respectivo para horizonte visual e velocidades de navegação variantes no tempo além de análise de robustez.

\section{REFERÊNCIAS}

Oualid Araar, Nabil Aouf, and Ivan Vitanov. Vision based autonomous landing of multirotor uav on moving platform. Journal of Intelligent \& Robotic Systems, 85 (2):369-384, Feb 2017.
A. Das, O. Naroditsky, Z. Zhu, S. Samarasekera, and R. Kumar. Robust visual path following for heterogeneous mobile platforms. In 2010 IEEE International Conference on Robotics and Automation, pages 24312437, May 2010. doi: 10.1109/ROBOT.2010.5509699.

Nuno de Oliveira and Lorenz T Biegler. Constraint handing and stability properties of model-predictive control. AIChE journal, 40(7):1138-1155, 1994.

G. W. Huang, C. H. Wu, C. C. Lai, S. T. Pan, C. H. Tsai, S. J. Lee, and C. S. Ouyang. Track model regression using genetic expression programming for visual-based path-following of mobile robots. In 2016 Asia-Pacific Conference on Intelligent Robot Systems (ACIRS), pages 47-51, July 2016. doi: 10.1109/ACIRS. 2016.7556186.

F. Ke, Z. Li, and C. Yang. Robust tube-based predictive control for visual servoing of constrained differentialdrive mobile robots. IEEE Transactions on Industrial Electronics, 65(4):3437-3446, April 2018. ISSN 02780046. doi: 10.1109/TIE.2017.2756595.

D. Q. Mayne and P. O. M. Scokaert J. B. Rawlings, C. V. Rao. Constrained model predictive control: Stability and optimality. Automatica, 36(6):789-814, June 2000.

R. O. ; Conceicao A. G. S. Ribeiro, T. T.; Fernandes. Seguimento de caminhos visuais baseado em nmpc para robós diferenciais. In XXI Congresso Brasileiro de Automática - CBA2016, 2016a.

R. O. ; Conceicao A. G. S. Ribeiro, T. T.; Fernandes. Garantia de estabilidade e factibilidade de controladores nmpc para o seguimento de caminhos visuais. In $X X I$ Congresso Brasileiro de Automática - CBA2016, 2016b.

Tiago Ribeiro and Andre Scolari Conceicao. Nonlinear model predictive visual path following control to autonomous mobile robots. Journal of Intelligent \& Robotic Systems, 07 2018. doi: 10.1007/s10846-018-0896-3.

R. T. Rodrigues, M. Basiri, A. P. Aguiar, and P. Miraldo. Low-level active visual navigation: Increasing robustness of vision-based localization using potential fields. IEEE Robotics and Automation Letters, 3(3):2079-2086, July 2018. doi: 10.1109/LRA.2018.2809628.

P.O.M. Scokaert, D.Q. Mayne, and J.B. Rawlings. Suboptimal model predictive control (feasibility implies stability). Automatic Control, IEEE Transactions on, 44(3):648-654, Mar 1999. ISSN 0018-9286. doi: 10.1109/ 9.751369 .

P. Spellucci. An sqp method for general nonlinear programs using only equality constrained subproblems. In Mathematical Programming, volume 82, pages $413-448$, 1998.

S. K. v. Es and T. D. Barfoot. Being in two places at once: Smooth visual path following on globally inconsistent pose graphs. In 2015 12th Conference on Computer and Robot Vision, pages 54-61, June 2015. doi: 10.1109/ CRV.2015.17.

X. Zhang, R. Wang, Y. Fang, B. Li, and B. Ma. Acceleration-level pseudo-dynamic visual servoing of mobile robots with backstepping and dynamic surface control. IEEE Transactions on Systems, Man, and Cybernetics: Systems, PP(99):1-11, 2017. ISSN 21682216. doi: 10.1109/TSMC.2017.2777897. 


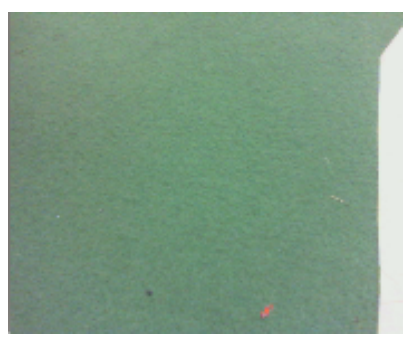

(a) Caminho (modo N).

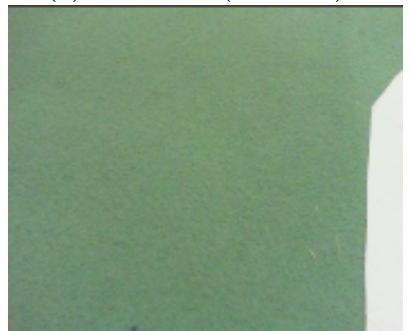

(d) Caminho (modo GE).

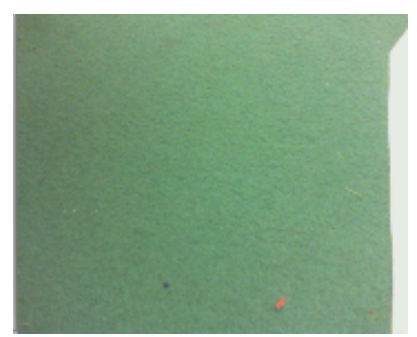

(g) Caminho (modo GF).

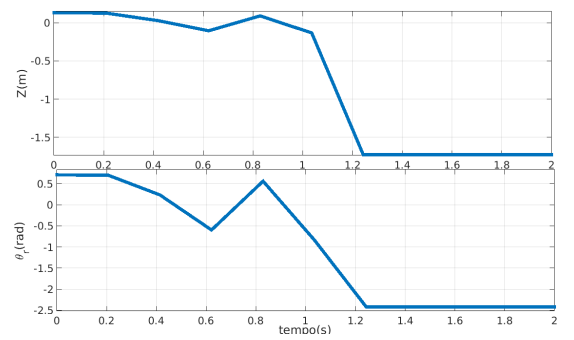

(b) Erros de seguimento (modo N).

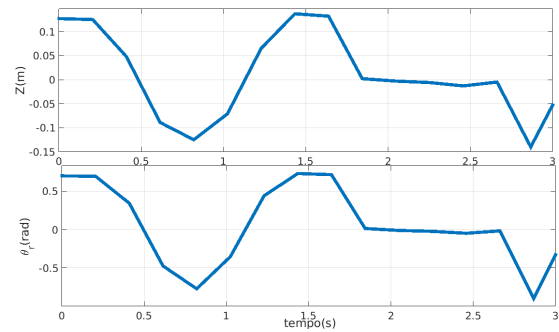

(e) Erros de seguimento (modo GE).

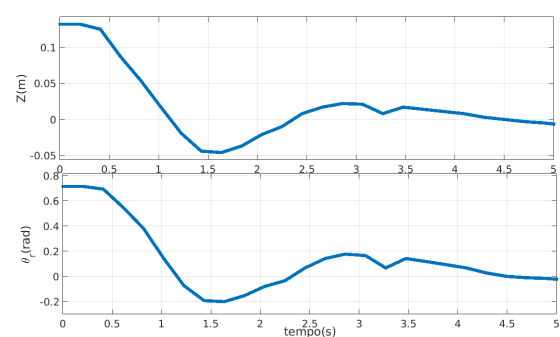

(h) Erros de seguimento (modo GF).

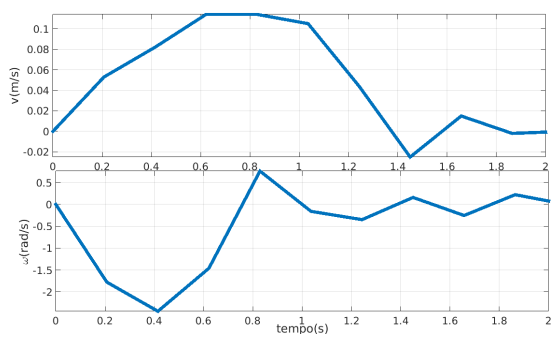

(c) Esforços de controle (modo N).

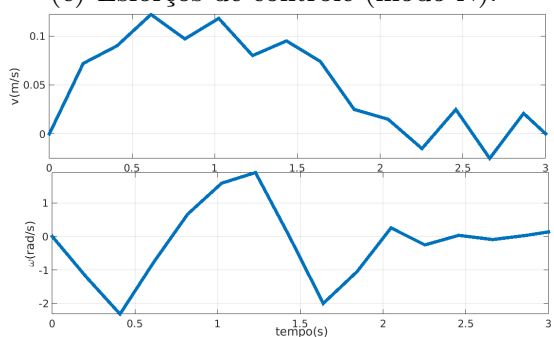

(f) Esforços de controle (modo GE).

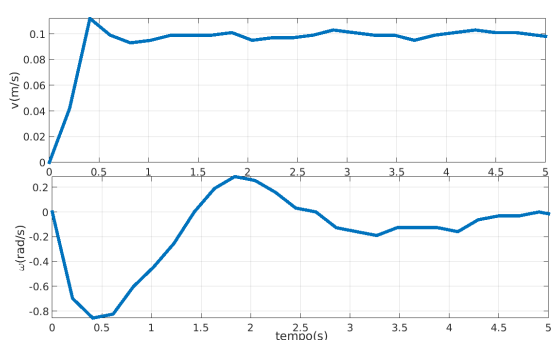

(i) Esforços de controle (modo GF).

Figura 3. Caminho vertical no plano da imagem - $N M P C$ com garantia de estabilidade $-v=0,1 \mathrm{~m} / \mathrm{s}$.

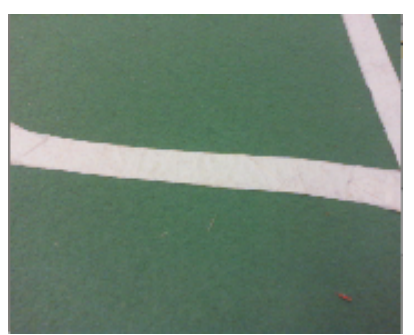

(a) Caminho (modo N).

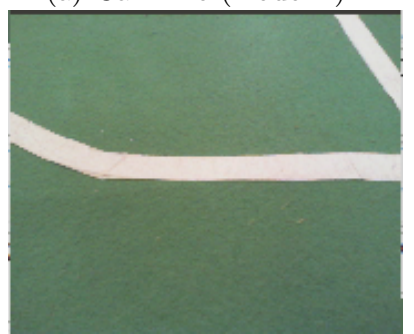

(d) Caminho (modo GE).

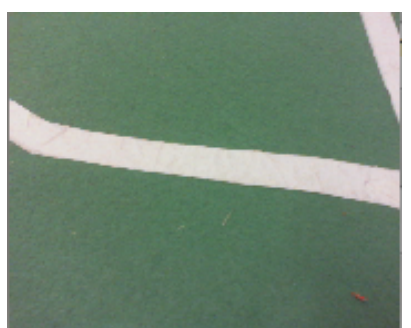

(g) Caminho (modo GF).

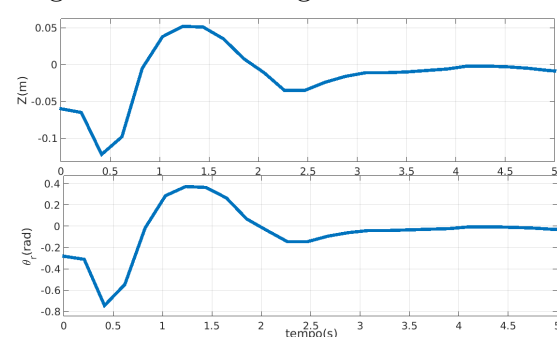

(b) Erros de seguimento (modo N).

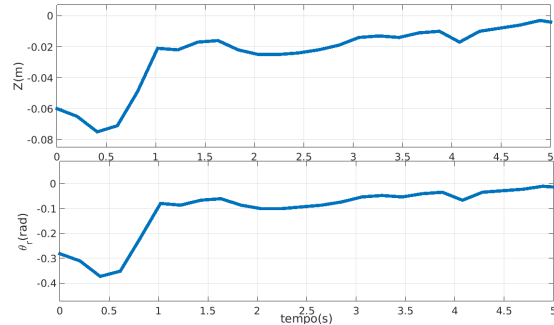

(e) Erros de seguimento (modo GE).

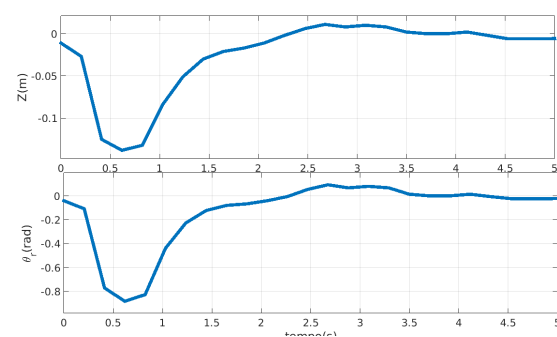

(h) Erros de seguimento (modo GF).

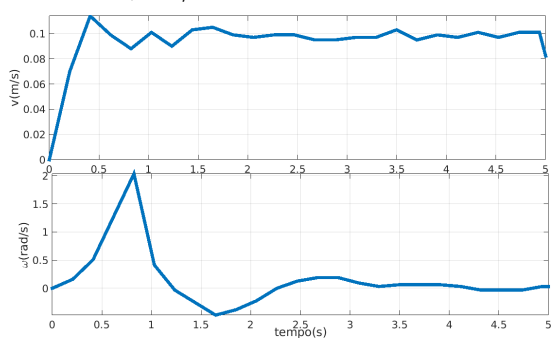

(c) Esforços de controle (modo N).

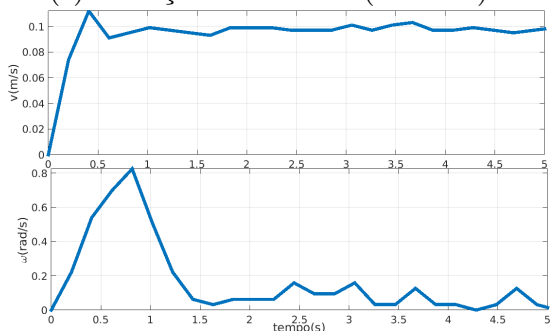

(f) Esforços de controle (modo GE).

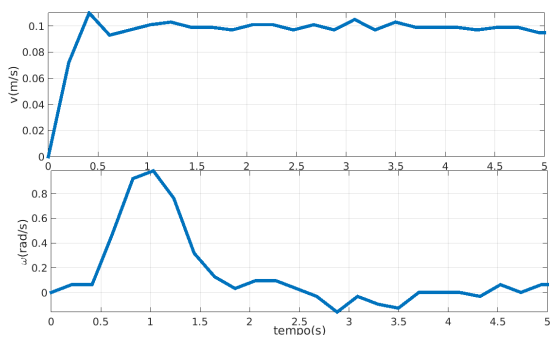

(i) Esforços de controle (modo GF).

Figura 4. Caminho horizontal no plano da imagem - $N M P C$ com garantia de factibilidade $-v=0,1 \mathrm{~m} / \mathrm{s}$. 